\title{
On the Intrinsic Population of the Lowest Triplet State of Thymine
}

\author{
Juan José Serrano-Pérez, Remedios González-Luque, \\ Manuela Merchán, Luis Serrano-Andrés* \\ Instituto de Ciencia Molecular, Universitat de València, \\ Apartado 22085, ES-46071 Valencia, Spain
}

\section{Supporting Information}

* To whom correspondence should be addressed. E-mail: Luis.Serrano@uv.es. 


\section{Additional computational details.}

All calculations reported in the present contribution have been performed using the CASPT2//CASSCF protocol, in which geometry optimizations, including minima, surface crossings, and minimum energy paths (MEP) were carried out at the multiconfigurational CASSCF level, whereas electronic energy calculations used the second-order multiconfigurational perturbation approach, CASPT2. ${ }^{1,2,3,4}$ MEP optimizations employed different active spaces: $\operatorname{CASSCF}(10,11)$, $\operatorname{CASSCF}(12,9)$, and $\operatorname{CASSCF}(10,8)$ for MEPs on ${ }^{1}\left(\pi \pi^{*}\right),{ }^{1}\left(\mathrm{n}_{\mathrm{O}} \pi^{*}\right)$, and ${ }^{3}\left(\pi \pi^{*}\right)$, respectively. Whereas the full $\pi \pi^{*}$ active space comprises ten electrons in eight orbitals, three additional extra correlating $\pi^{*}$ MOs, which had to be added to compute the MEP on ${ }^{1}\left(\pi \pi^{*}\right)$ in order to balance dynamic correlation effects. The nature of the orbitals does not essentially change along the optimization process. The final CASPT2 calculations at each of the optimized structures used an active space comprising 14 electrons distributed in 10 orbitals. A more detailed discussion about the choice of the active space and its consequences can be found in our previous study on DNA pyrimidine nucleobases. ${ }^{5}$ The employed basis set was ANO-S C,N,O[3s2p1d]/H[2s1p]. The standard zeroth-order Hamiltonian was employed in the CASPT2 calculations, which include an imaginary level-shift correction of 0.2 au in order to avoid the presence of intruder states. ${ }^{6}$ All calculations used the MOLCAS-6.0 set of programs. ${ }^{7}$

The MEP has been built as steepest descendent paths in a procedure ${ }^{8}$ which is based on a modification of the projected constrained optimization (PCO) algorithm of Anglada and Bofill ${ }^{9}$ and follows the Müller-Brown approach. ${ }^{10}$ Each step requires the minimization of the PES on a hyperspherical cross section of the PES centered on the initial geometry and characterized by a predefined radius. The optimized structure is taken as the center of a new hypersphere of the same radius, and the procedure is iterated until the bottom of the energy surface is reached. Mass-weighted coordinates are used, therefore the MEP coordinate corresponds to the so-called Intrinsic Reaction Coordinate (IRC), measured in au, that is, bohr $(\mathrm{amu})^{1 / 2}$. The full procedure is currently implemented in the MOLCAS-6.0 package $^{7}$ and its technical description has been published elsewhere. ${ }^{8}$ As mentioned above, at each optimized point of the MEP, CASPT2//CASSCF $(14,10)$ calculations were performed for the states of interest. The MEP search showed to be insensitive to different selections of the step length.

In this contribution conical intersection searches were performed using the restricted Lagrange multipliers technique as included in the MOLCAS-6.0 package ${ }^{7}$ in which the lowest-energy point was obtained under the restriction of degeneracy between the two considered states. ${ }^{8}$

From the calculated CASSCF transition dipole moments (TDM) and the CASPT2 excitation energies, the radiative lifetimes have been estimated by using the Strickler-Berg relationship, ${ }^{11}$ as 
explained elsewhere. ${ }^{12}$ The spin-orbit coupling (SOC) strength between selected states was computed within the AMFI framework obtaining as the length of the spin-orbit coupling vector with the algorithms implemented in the MOLCAS- $6.0^{7}$ quantum-chemistry program, as described previously. ${ }^{13}$ A CASSCF wave function averaged over seven singlet and seven triplet states was used.

Table SI1 compiles selected bond lengths in thymine at different optimized geometries. Figure SI1 displays the evolution of the MEP from the singlet-triplet crossing (STC) $\left({ }^{3} \mathrm{n}_{\mathrm{O}} \pi^{*} /{ }^{1} \pi \pi^{*}\right)_{\mathrm{STC}}$ reaching the minimum $\left({ }^{3} n_{0} \pi^{*}\right)_{\min }$. Figure SI2 includes the evolution of the MEP from the conical intersection $\left({ }^{3} n_{O} \pi /^{3} \pi \pi^{*}\right)_{\mathrm{CI}}$, practically coincident with the minimum $\left({ }^{3} \mathrm{n}_{\mathrm{O}} \pi^{*}\right)_{\min }$, to the minimum $\left({ }^{3} \pi \pi^{*}\right)_{\min }$. Figure SI3 displays the evolution of the MEP from the STC $\left(\mathrm{gs} /{ }^{3} \pi \pi^{*}\right)_{\mathrm{STC}}$ reaching the minimum $\left({ }^{3} \pi \pi^{*}\right)_{\min }$. In all cases seven singlet and seven triplet states were included in the calculations, that were performed at the CASPT2//CASSCF $(14,10)$ level. Figure SI4 compiles the structures of the optimized singular points reported in the present research.

Reference 7 of this Supporting Information corresponds to the complete Ref. 20 of the paper.

\section{References}

(1) Andersson, K.; Malmqvist, P.-Å., Roos, B. O. J. Chem. Phys. 1992, 96, 1218.

(2) Roos, B. O.; Andersson, K.; Fülscher, M. P.; Malmqvist, P.-Å.; Serrano-Andrés, L.; Pierloot, K.; Merchán, M. Adv. Chem. Phys. 1996, 93, 219.

(3) Merchán, M.; Serrano-Andrés, L.; Fülscher, M. P.; Roos, B. O. In Recent Advances in Multireference Methods, Hirao, H.; Ed.,World Scientific, Singapore, 1999.

(4) Merchán, M.; Serrano-Andrés, L. In: Computational Photochemistry; Olivucci, M. Ed; Elsevier, Amsterdam, 2005

(5) Merchán, M.; González-Luque, R.; Climent, T.; Serrano-Andrés, L.; Rodríguez, E.; Reguero, M.; Peláez, D. J. Phys. Chem.B 2006, 110, 26471.

(6) Forsberg, J.; Malmqvist, P.-Å. Chem. Phys. Lett. 1997, 274, 196.

(7) Andersson, K.; Barysz, M.; Bernhardsson, A.; Blomberg, M. R. A.; Carissan, Y.; Cooper, D. L.; Cossi, M.; Fülscher, M. P.; Gagliardi, L.; de Graaf, C.; Hess, B.; Hagberg, G.; Karlström, G.; Lindh, R.; Malmqvist, P.-Å.; Nakajima, T.; Neogrády, P.; Olsen, J.; Raab, J.; Roos, B. O.; Ryde, U.; Schimmelpfennig, B.; Schütz, M.; Seijo, L.; Serrano-Andrés, L.; Siegbahn, P. E. M.; Stålring, J.; Thorsteinsson, T.; Veryazov, V.; Widmark, P.-O. MOLCAS, version 6.0; Department of Theoretical Chemistry, Chemical Centre, University of Lund: Lund, Sweden, 2004.

(8) De Vico, L.; Olivucci, M.; Lindh, R. J. Chem. Theory Comp. 2005, 1, 1029.

(9) Anglada, J. M.; Bofill, J.M. J. Comput. Chem. 1997, 18, 992.

(10) Müller, K.; Brown, L. D. Theor. Chim. Acta. 1979, 53, 75.

(11) Strickler, S. J.; Berg, R. A. J. Chem. Phys. 1962, 37, 814.

(12) Rubio-Pons, O.; Serrano-Andrés, L.; Merchán, M. J. Phys. Chem. A 2001, 105, 9664-9676.

(13) Merchán, M.; Serrano-Andrés, L.; Robb, M. A.; Blancafort, L. J. Am. Chem. Soc. 2005, 127, 1820. 
Table SI1. Selected internal coordinates at the singular points computed for thymine.

\begin{tabular}{|c|c|c|c|c|c|c|c|c|}
\hline parameters $^{\mathrm{a}}$ & $\mathrm{gs}^{\mathrm{b}}$ & ${ }^{1}\left(\pi, \pi^{*}\right)^{b}$ & ${ }^{1}\left(\mathrm{n}_{\mathrm{O}}, \pi^{*}\right)^{\mathrm{c}}$ & $\mathrm{CI}(\mathrm{S})^{\mathrm{d}}$ & ${ }^{3}\left(\pi, \pi^{*}\right)^{b}$ & ${ }^{3}\left(\mathrm{n}_{\mathrm{O}}, \pi^{*}\right)^{\mathrm{c}}$ & $\mathrm{CI}(\mathrm{T})^{\mathrm{e}}$ & STC $^{f}$ \\
\hline $\mathrm{R}\left(\mathrm{N}_{1}-\mathrm{C}_{2}\right)$ & 1.370 & 1.413 & 1.369 & 1.445 & 1.382 & 1.369 & 1.369 & 1.384 \\
\hline $\mathrm{R}\left(\mathrm{C}_{2}-\mathrm{N}_{3}\right)$ & 1.378 & 1.338 & 1.378 & 1.365 & 1.384 & 1.387 & 1.387 & 1.388 \\
\hline $\mathrm{R}\left(\mathrm{N}_{3}-\mathrm{C}_{4}\right)$ & 1.387 & 1.430 & 1.396 & 1.449 & 1.396 & 1.399 & 1.399 & 1.393 \\
\hline $\mathrm{R}\left(\mathrm{C}_{4}-\mathrm{C}_{5}\right)$ & 1.476 & 1.399 & 1.367 & 1.474 & 1.455 & 1.381 & 1.381 & 1.497 \\
\hline $\mathrm{R}\left(\mathrm{C}_{5}-\mathrm{C}_{6}\right)$ & 1.346 & 1.502 & 1.406 & 1.448 & 1.513 & 1.393 & 1.393 & 1.509 \\
\hline $\mathrm{R}\left(\mathrm{C}_{5}-\mathrm{C}_{7}\right)$ & 1.503 & 1.500 & 1.510 & 1.558 & 1.494 & 1.510 & 1.509 & 1.514 \\
\hline $\mathrm{R}\left(\mathrm{C}_{6}-\mathrm{N}_{1}\right)$ & 1.386 & 1.334 & 1.404 & 1.311 & 1.397 & 1.401 & 1.400 & 1.402 \\
\hline $\mathrm{R}\left(\mathrm{C}_{2}-\mathrm{O}_{2}\right)$ & 1.198 & 1.206 & 1.200 & 1.190 & 1.197 & 1.199 & 1.199 & 1.194 \\
\hline $\mathrm{R}\left(\mathrm{C}_{4}-\mathrm{O}_{4}\right)$ & 1.201 & 1.237 & 1.365 & 1.194 & 1.206 & 1.361 & 1.360 & 1.199 \\
\hline$<\left(\mathrm{N}_{1} \mathrm{C}_{2} \mathrm{~N}_{3}\right)$ & 114.1 & 115.2 & 114.7 & 114.0 & 115.0 & 115.0 & 115.0 & 114.8 \\
\hline$<\left(\mathrm{C}_{2} \mathrm{~N}_{3} \mathrm{C}_{4}\right)$ & 127.2 & 124.9 & 122.2 & 123.8 & 124.5 & 120.7 & 120.7 & 126.7 \\
\hline$<\left(\mathrm{N}_{3} \mathrm{C}_{4} \mathrm{C}_{5}\right)$ & 115.4 & 117.7 & 123.0 & 112.3 & 115.1 & 122.2 & 122.3 & 112.8 \\
\hline$<\left(\mathrm{C}_{4} \mathrm{C}_{5} \mathrm{C}_{6}\right)$ & 117.6 & 119.3 & 116.1 & 110.4 & 115.9 & 116.3 & 116.3 & 109.7 \\
\hline$<\left(\mathrm{C}_{5} \mathrm{C}_{6} \mathrm{~N}_{1}\right)$ & 122.6 & 115.5 & 119.2 & 119.0 & 111.9 & 119.7 & 119.7 & 109.2 \\
\hline$<\left(\mathrm{C}_{7} \mathrm{C}_{5} \mathrm{C}_{4}\right)$ & 118.4 & 120.5 & 121.9 & 105.9 & 121.6 & 121.6 & 121.6 & 114.7 \\
\hline$<\left(\mathrm{C}_{6} \mathrm{~N}_{1} \mathrm{C}_{2}\right)$ & 123.3 & 127.5 & 124.8 & 117.3 & 120.9 & 124.3 & 124.3 & 118.5 \\
\hline$<\left(\mathrm{O}_{2} \mathrm{C}_{2} \mathrm{~N}_{1}\right)$ & 123.2 & 117.3 & 123.3 & 118.7 & 122.7 & 123.2 & 123.2 & 123.0 \\
\hline$<\left(\mathrm{O}_{4} \mathrm{C}_{4} \mathrm{~N}_{3}\right)$ & 120.5 & 115.0 & 112.5 & 116.7 & 120.3 & 112.0 & 111.9 & 121.3 \\
\hline$<\left(\mathrm{C}_{7} \mathrm{C}_{5} \mathrm{C}_{4} \mathrm{C}_{6}\right)$ & 179.0 & 179.8 & 179.8 & 118.6 & 166.5 & 179.0 & 178.9 & 137.1 \\
\hline
\end{tabular}

${ }^{\mathrm{a}}$ Bonds lengths (R) in A and angles $(<)$ in degrees. See Figure 1 for atom labelling. ${ }^{b} \mathrm{CASSCF}(10,8) / \mathrm{ANO}-\mathrm{S} \mathrm{C}, \mathrm{N}, \mathrm{O}[3 \mathrm{~s} 2 \mathrm{p} 1 \mathrm{~d}] / \mathrm{H}[2 \mathrm{~s} 1 \mathrm{p}]$ level. ${ }^{\mathrm{c}} \mathrm{CASSCF}(12,9) / \mathrm{ANO}-\mathrm{S}$

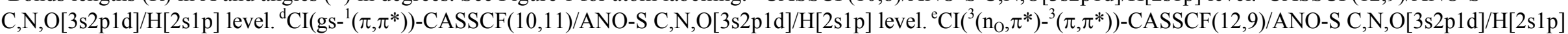
level. ${ }^{\mathrm{STC}}\left(\mathrm{GS}^{3}{ }^{3}\left(\pi, \pi^{*}\right)\right)-\mathrm{CASSCF}(10,8) / \mathrm{ANO}-\mathrm{S} \mathrm{C}, \mathrm{N}, \mathrm{O}[3 \mathrm{~s} 2 \mathrm{p} 1 \mathrm{~d}] / \mathrm{H}[2 \mathrm{~s} 1 \mathrm{p}]$ level. 


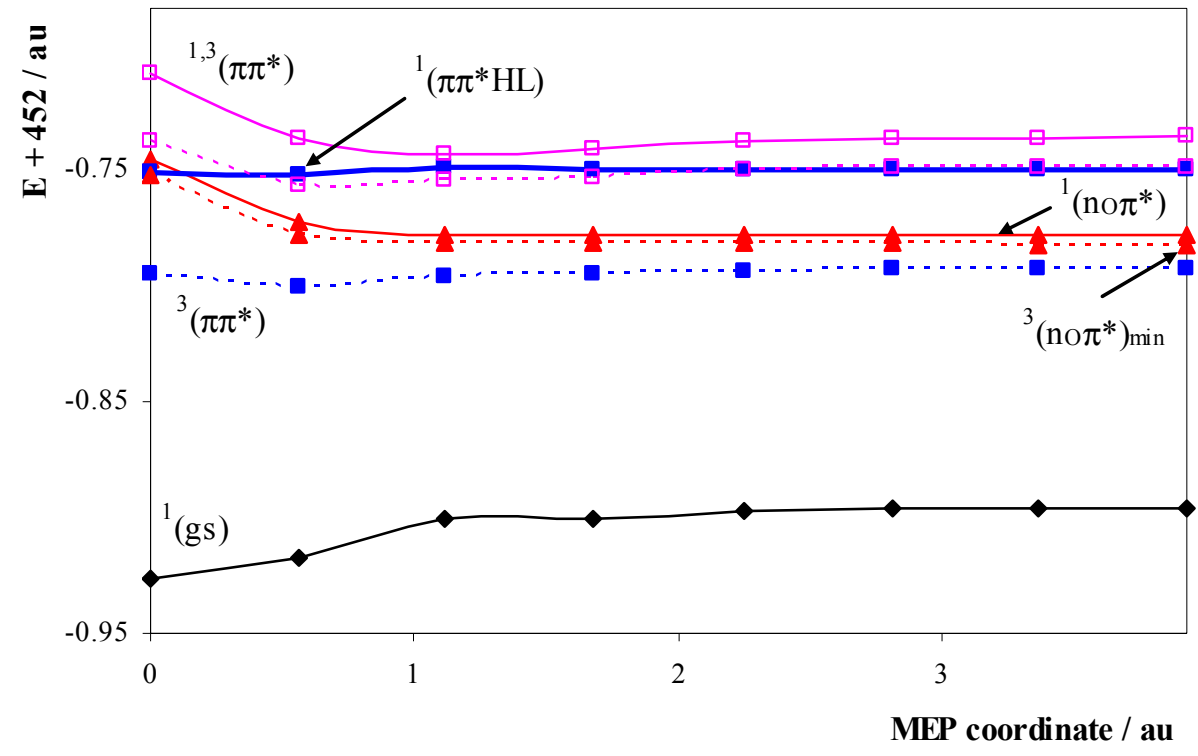

Figure SI1. Evolution in thymine of the minimum energy path (MEP) from the singlet-triplet crossing $\left({ }^{3} \mathrm{n}_{\mathrm{O}} \pi * /{ }^{1} \pi \pi^{*}\right)_{\mathrm{STC}}$ reaching the minimum $\left({ }^{3} \mathrm{n}_{\mathrm{O}} \pi *\right)_{\min }$. CASPT2//CASSCF $(14,10) / \mathrm{ANO}-\mathrm{S}$ $\mathrm{C}, \mathrm{N}, \mathrm{O}[3 \mathrm{~s} 2 \mathrm{p} 1 \mathrm{~d}] / \mathrm{H}[2 \mathrm{~s} 1 \mathrm{p}]$ level. 


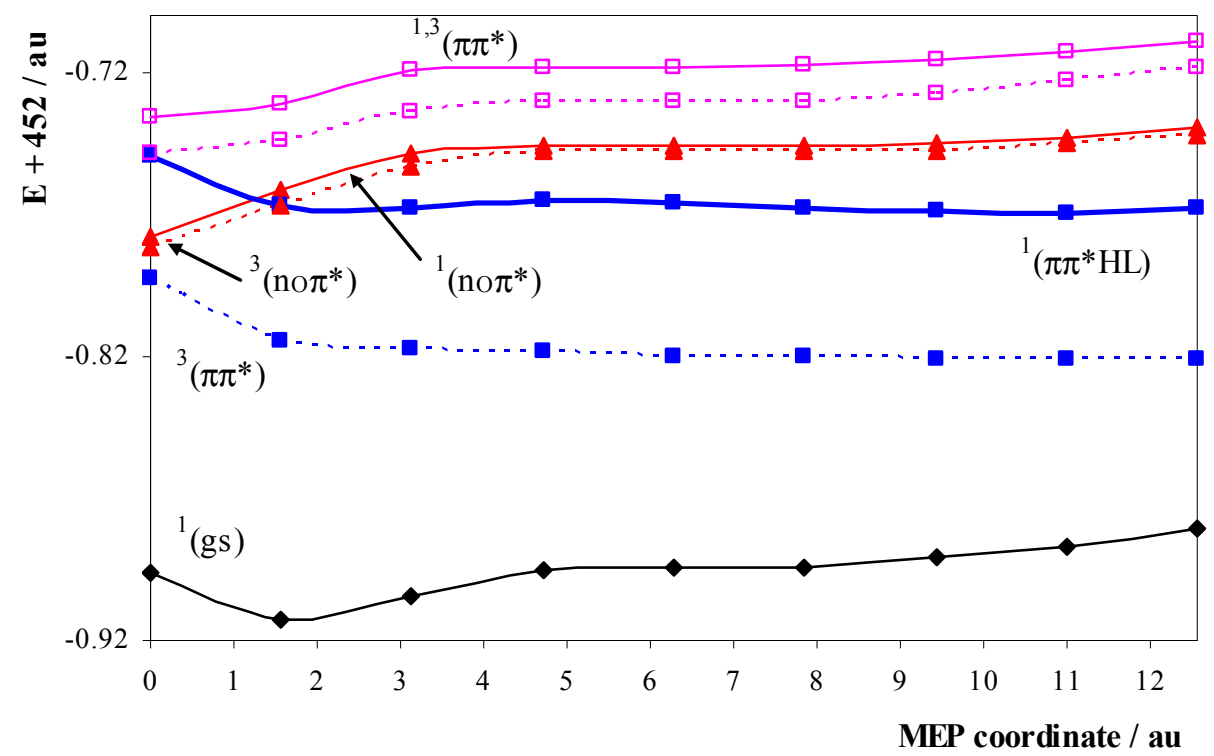

Figure SI2. Evolution in thymine of the minimum energy path (MEP) along the ${ }^{3}\left(\pi \pi^{*}\right)$ state from the conical intersection $\left({ }^{3} n_{\mathrm{O}} \pi /^{3} \pi \pi^{*}\right)_{\mathrm{CI}}$ to the minimum $\left({ }^{3} \pi \pi^{*}\right)_{\min }$. CASPT2//CASSCF $(14,10) / \mathrm{ANO}-\mathrm{S}$ $\mathrm{C}, \mathrm{N}, \mathrm{O}[3 \mathrm{~s} 2 \mathrm{p} 1 \mathrm{~d}] / \mathrm{H}[2 \mathrm{~s} 1 \mathrm{p}]$ level of calculation. 


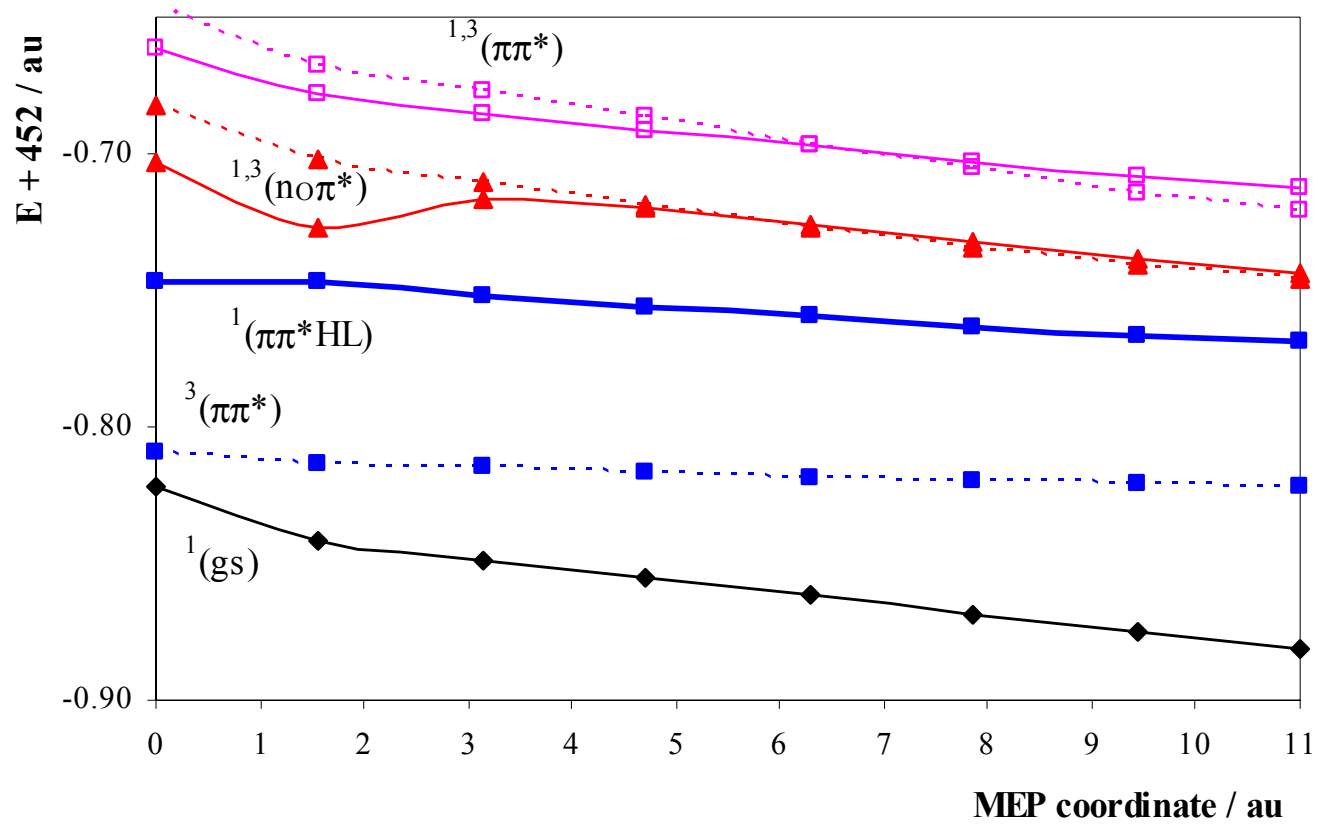

Figure SI3. Evolution in thymine of the minimum energy path (MEP) along the ${ }^{3}\left(\pi \pi^{*}\right)$ state from the singlet-triplet crossing $\left(\mathrm{gs}^{3} \pi \pi^{*}\right)_{\mathrm{STC}}$ reaching the minimum ${ }^{3}\left(\pi \pi^{*}\right)_{\min }$. Level of calculation: CASPT2//CASSCF $(14,10) / A N O-S$ C,N,O[3s2p1d]/H[2s1p]. 

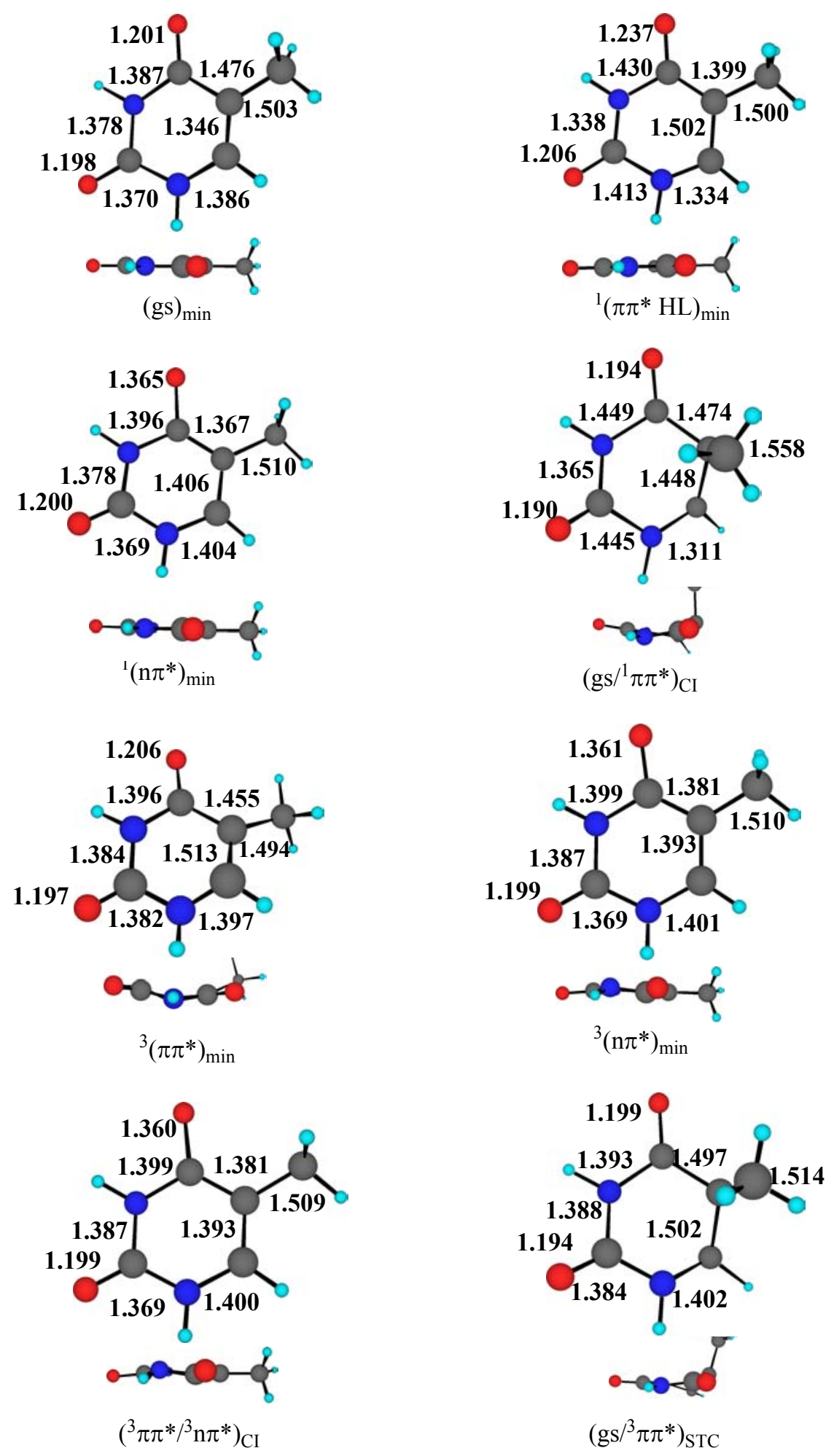

Figure SI4. Structures in the singular points computed for thymine. 